\title{
Increasing midwives' ethical competence: a European educational and practice development project
}

\author{
Stephan Oelhafen ${ }^{1}$ (D) Ursula Hölzli ${ }^{1}$. \\ Marjatta Häsänen ${ }^{2}$ - Annely Kärema ${ }^{3}$. \\ Minni-Triin Kasemets ${ }^{3}$ • Irena Bartels ${ }^{4}$. \\ Aet Maarja Leberecht ${ }^{4}$ - Marjo Kauppila ${ }^{5}$. \\ Irmeli Järvilehto-Impivaara $^{5} \cdot$ Mari Berglund $^{2}$
}

Published online: 22 February 2017

C) Springer International Publishing AG 2017

\begin{abstract}
Midwives, like other health professionals, are confronted with ethical issues on a daily basis and acting ethically is a core competence within professional conduct. In midwifery in particular, the complexity of ethical problems is increasing e.g., due to new medical options in diagnostics and reproductive medicine, the increasing diversity of life styles or the high number of preterm births. The main purpose of the current project was to develop effective interventions to increase midwives' ethical competence in educational and practical settings. To reach this goal, we conducted 43 individual semi-structured and 6 focus group interviews with midwives, lecturers and midwifery students to explore their views on ethics education and the support needed in practice to develop ethical competence. Based on the analysis of these interviews, a set of 15 interventions was designed and piloted in educational and clinical settings. The piloted interventions were then evaluated with an online questionnaire. Results from the interviews, the online questionnaire as well as the lessons learnt from piloting the interventions and the current literature on ethics education were integrated in a spiral curricular model for increasing
\end{abstract}

Electronic supplementary material The online version of this article (doi:10.1007/s40889-017-0033-3) contains supplementary material, which is available to authorized users.

Stephan Oelhafen

stephan.oelhafen@bfh.ch

1 Health Division, Bern University of Applied Sciences, Bern, Switzerland

2 Faculty of Health and Well-being, Turku University of Applied Sciences, Turku, Finland

3 Womens' Clinic, East-Tallinn Central Hospital, Tallinn, Estonia

4 Chair of Midwifery, Tallinn Health Care College, Tallinn, Estonia

5 Department of Obstetrics and Gynecology, Turku University Hospital, Turku, Finland 
midwives' ethical competence. This model, called MidEthics, covers essential competences for midwives to be obtained in training and in clinical practice, and should inform curriculum development and policy makers.

Keywords Moral competence · Ethical competence · Midwives · Moral issues · Educational model

\section{Introduction}

Ethical competence is defined as the ability to solve a moral problem by reflecting and acting in a manner which considers the values, convictions, emotions and needs of all persons involved (Kavathatzopoulos 2003). The necessary skills are: possessing ethical knowledge, being able to perceive the problem as such and reflecting on it critically, having the will and courage to address it, and, consequently, acting ethically (Gallagher 2006). It is of major importance for client-centered care and, along with professional knowledge and skills, forms the basis of professional conduct for midwives. Midwifery care is believed to be complex by nature because at least two persons, mother and child, with potentially conflicting needs and values have to be considered. Additionally, there are indications that this complexity is increasing for a number of reasons. First, the diversity of societies, cultures and life styles is rising, due, in part, to migration and high numbers of refugees. Second, medical options such as prenatal diagnostics or assisted reproduction demand new skills of midwives on the one hand and alter the expectations of parents-to-be on the other. Third, working in multiprofessional teams whose members may have varying perspectives on situations and differing personal or professional values creates challenges for care providers. Finally, dealing with specific problems such as preterm births, substance abuse or domestic violence is particularly demanding.

In all circumstances midwives are expected to protect human dignity, to provide care with respect, and support women and their families in their decision making (International Confederation of Midwives 2014b). The foundation of moral decisions and actions are sound professional knowledge and skills (International Confederation of Midwives 2014a). Further guidance is found in professional codes, such as the International Code of Ethics for Midwives (International Confederation of Midwives 2014a), in ethics theory, and in reflection on personal values and attitudes. The midwife's ability to follow this guidance and make autonomous decisions is highly dependent on the laws and regulations of a country, the institutional context and personal factors (Marshall 2005). Historically, the hospital birth rate has increased steadily in high- and middle-income countries throughout the 20th century, limiting both the midwives' and the women's autonomy (Marshall 2005). In the clinical context, the midwife is dependent on an institutional hierarchy, institutional guidelines and particularly medical prescriptions. At the same time, the women's freedom of choice regarding place of birth is restricted (Olsen and Clausen 2012) and at least from a midwife's perspective, women are exposed to a paternalistic decision making model (Cahill 2001; Noseworthy et al. 2013). Therefore, normalizing birth and de-medicalizing 'low-risk' pregnancies could help regaining women's and midwives’ autonomy. 
To support the development of ethical competence in health care, educational institutions generally offer formal training and in some hospitals ethics boards may be available for case discussions. However, reflection is needed on how to successfully transfer theoretical knowledge, rules and codes into practice (Bolmsjö et al. 2006). Current ethics education has been criticized for often being based on extreme dilemma situations, such as induced abortion in advanced pregnancy or life support for extremely premature infants (Liaschenko et al. 2006). Such situations are undoubtedly morally challenging for the care providers, yet, they do not represent their daily work, and reflecting on them does not give sufficient guidance for everyday practice (Liaschenko et al. 2006). Acting ethically is required at all times and is not restricted to moral dilemmas. Most everyday situations include topics such as informed decision making, respecting the autonomy of clients even for routine interventions, or protecting intimacy and privacy (Bolmsjö et al. 2006). Practitioners need skills to master all situations ethically, independent of their nature (Molewijk et al. 2008).

The current project was initiated to develop a set of interventions in the clinical and educational setting in order to create a model for education and practice that would increase the ethical competence of midwives facing today's clinical challenges. With the international collaboration of partners from Turku (Finland), Tallinn (Estonia) and Bern (Switzerland), different cultural and societal backgrounds were present. This enabled insights into various perspectives and demands in the development of the model.

This article describes all the project phases carried out until a model called MidEthics could be formulated and presented here. The exploration includes an assessment of the perspectives on ethics education among lecturers, practitioners and students, as well as the support given to and/or needed by midwives to improve their own and their students' ethical competence in practice. The pilot phase describes the interventions designed for and tested in academic and practical settings. The evaluation reveals the interventions found to be most effective in improving ethical competence. Finally, the MidEthics model generated by the data and knowledge gained, based on current literature and a competence framework, is described and discussed. The step-by-step contingent process, as well as its outcomes, is presented here in the same order.

\section{Exploration}

The project started in all participating partner sites with an exploration phase to investigate how students and midwives are supported in increasing their ethical competence in the educational program and in clinical practice. Also, we wanted to assess students' and practicing midwives' experiences in their individual contexts. Therefore a qualitative approach with a combination of individual and group interviews was chosen to cover a large range of experiences. The comprehension of ethical problems has been shown to be dependent on context and profession (Barandun Schäfer et al. 2015), so we therefore specifically wanted to grasp the meaning of problems in being ethical from a midwife's point of view. Overall, the target during this phase was to generate ideas and inform the following pilot phase of interventions to improve the current situation. 


\section{Method of exploration phase}

A large variety of methods was chosen to cover a wide range of experiences in the educational and clinical context. For that purpose, 43 semi-structured individual interviews, 6 focus group interviews and a survey with 36 participants were conducted by midwifery lecturers, practicing midwives and supervised midwifery bachelor students. The interviews were conducted in the appropriate context, i.e., at midwifery school or at different obstetrics and gynecological wards in the clinics. Interview participants were midwifery students, lecturers, teaching or practicing midwives and nurses working in the same environment. Most participants were recruited as a convenience sample and a few were sampled purposefully because they held key positions in their particular domains. An ethics committee approval was not necessary as we interviewed only students or health professionals and we did not process or had access to any patient related data.

Whereas context, sampling strategy and interview methodology differed among the five partner sites, an English interview guide served as a common basis to create Finnish, Estonian and German interview guides, which were then adapted to the specific educational and practical context. These interview guides covered topics such as current ethics education, students' problems in clinical practice and the type of ethical problems experienced in clinical midwifery practice. All interview protocols were content analyzed in the original language. Themes were first created on the level of individual and then categorized on an inter-individual level within each group of participants, i.e., students or health professionals in their respective discipline or clinical division. The synthesized results were then translated into English and discussed in the working group of all partners. In general, we understand the experiences of each participant to be unique in a constructivist manner. However, for the purpose of the project, we aggregated individual responses and experiences from all partner sites intentionally to be able to use them for the subsequent development of pilot interventions, even though not all topics are relevant for each institution.

\section{Exploration results}

When midwifery students start their educational program, some of them seem to have a rather narrow understanding on what ethics is. They seem to equate ethics with having one's own individual moral system. Accordingly, some consider moral problems as a problem for themselves alone. However, teachers stress the importance of reflecting on their own behavior, convictions and personal values. If individuals' own system of morals and beliefs is not reflected upon, the autonomy of the client is not guaranteed if it conflicts with the midwife's personal values. Introductory lectures help to raise awareness on the topic of ethics in general, but without clinical experience, the theory remains abstract. Thus, midwifery students seem to have good theoretical knowledge, but they have problems in transferring and applying that knowledge in clinical situations. They have difficulties in understanding the core of a moral problem and how to name and frame it. The students themselves believe that case discussions and problem-based learning tutorials with cases are more helpful. However, several lecturers and midwives stressed that the cases need to be realistic, less extreme, and in the best case personally experienced by the students. Still, many students and midwives 
think that the influence of education on ethical competence is limited, and that mainly clinical experience is important.

Both students and teachers reported that when students do their clinical assignment for the first time, they realize that there is a large theory practice gap, e.g., between the ideal of a self-determined physiological birth and clinical reality; a lack of personal resources and a high workload often lead to ethical problems. Contrary to what they learnt in training, client autonomy seems to be limited, e.g., when a woman is against the induction of labor, and many decisions seem to be hierarchically driven as opposed to evidence-based. In this constellation, some students feel helpless and wonder what it helps to know better if they cannot change anything about the current situation. On the other hand, from a midwife's perspective, students need more moral courage and should be able to ask critical questions. They also need good communication skills, which provide an essential foundation for behaving in an ethical manner. This includes communication of unpleasant news, confidential information or delicate issues.

The support students get from more experienced midwives is a critical element in increasing ethical competence in the clinical setting. In general, students think they are well supported by their mentoring midwives. The midwives themselves are aware that they act as role models, especially during patient contacts. Overall, case discussions seem to be very helpful for the students. They greatly appreciate it if challenging situations are briefed and debriefed, however time in some clinics is too limited to allow this. Many students stated that family and peer support is also an essential element enabling them to handle critical situations. The midwives, on the other hand, stated that they feel they need further education in ethics themselves in order to support midwifery students appropriately.

\section{Discussion of exploration}

The interviews conducted with midwifery students, lecturers, midwives and nurses provided important insights into the ethical competence required in practice. Many midwifery students or inexperienced practicing midwives realize that there is a wide gap between what they expected to be the ideal of caring and the clinical reality. The ever-present hierarchy could discourage them to feel they have no influence, even more so because a clinical procedure may be deeply rooted in the culture of an organization and therefore not easily changed (cf. Thompson 2004). On the other hand, there are moments when it is important to stand up for a woman in labor; therefore it is important to raise awareness on ethical issues. Students need to distinguish between moral wrongness and moral complexity (Monteverde 2014). When observing morally wrong behavior they need moral courage to raise their voice (Lachman 2007); they need to assess the risk of potential consequences and they need the right communication skills to address the issue, e.g., with their supervisor. In morally complex situations however, the right behavior is not obvious from a professional's point of view and various judgments are reasonable (Monteverde 2014). This is when theory and codes of ethics may help them to frame the problem and decide on it. However, the interviews with midwifery students clearly showed that ethical theory remains too abstract and they have problems applying that knowledge.

In any case, midwives' judgements should be based on professional values. The interviews with midwifery students suggest that they still rely heavily on their personal 
values and convictions. Therefore, learning to distinguish between personal and professional values is an essential requirement for acting in an ethical manner. This involves being self-aware, i.e., knowing one's own beliefs, convictions and prejudices (Thompson 2004). This required reflection on one's own values can be promoted with case discussions, e.g., with peers, or in guided discussions with the lecturer in combination with ethical theory. Whenever possible, the cases chosen to be discussed should be personally experienced and realistic everyday cases (Liaschenko et al. 2006).

Midwifery students and inexperienced midwives need additional support when working in a clinic. In general, the mentoring system was considered to be good by both regular midwives and students. One essential element is the briefing and debriefing of difficult situations, which obviously greatly depends on the available resources in the ward concerned. Mentoring midwives are aware of their responsibility to act as a role model for the students. However, especially younger mentors feel insecure about the right way to handle difficult situations, which demonstrates the necessity of the improvements we are striving for within this project.

\section{Pilot}

\section{Description of local interventions}

Based on the themes that emerged from the exploration, all partner institutions developed several interventions to increase ethical competence. These interventions differed in their thematic focus and were adjusted to the local context in terms of peculiarities of the Bachelor's program or the clinical situation. Altogether, a total of 15 interventions, i.e., teaching modules, discussion groups or seminars in the clinical setting, were developed for this project and evaluated.

At the Turku University of Applied Sciences and the Turku University Hospital, there was a marked focus on the development of new learning material and methods. Cases from midwifery practice were created and made available on an e-learning platform in the form of texts, illustrations and videos. Access was provided to midwifery students and midwives working in the clinic, facilitating different types of blended or self-paced learning. Also, an ethics day was held to raise awareness of ethical issues in general and test new participatory learning methods (e.g., sociodrama; McLaughlin Dorcas et al. 2006; Lindqvist 2005). In the clinical setting, two different discussion groups were offered for practicing midwives or students during their clinical practice. In both groups, everyday issues were discussed to stimulate ethical discussions within the team and to promote an analysis of personal and professional values. Also, expert lectures were arranged for the clinical setting that additionally covered ethical theories in the decision making process.

At the East-Tallinn Central Hospital, a seminar for regular midwives was introduced that combined an introductory lecture, e-learning based cases for self-study and case discussions. The focus was on active listening and self-reflection. Tallinn Health Care College introduced new teaching modules throughout the midwifery curriculum. In a two day seminar, introductory lectures were combined with discussions of cases from the students' clinical practice. The cases were also analyzed using the International Confederation of Midwives' (ICM) code of ethics. The discussions covered topics like 
role dependent communication, miscommunication in the workplace and self-reflection. In a series of problem-based learning tutorials (PBL), several typical moral issues were covered such as overcoming prejudice, e.g., in the case of a client having a different cultural background, actively integrating the needs and preferences of the partner or other family members and refusing service and referring a patient in the case of the midwife having deep moral opposition. Also, an ethics day with expert lectures and poster presentations was introduced.

At the Bern University of Applied Sciences, the teaching modules of the first and second year of the existing ethics curriculum for midwifery students were modified. In a first PBL module, students were confronted with a typical midwifery case that made them think and discuss their personal and professional values, which again were studied related to the ICM code of ethics. In another module before their first clinical assignment, students learnt about the gaps between theory and practice they could come across, the frustration or moral distress they might experience and possible coping strategies that could be applied (The 4 A's; American Association of Critical-Care Nurses 2004). After their first clinical assignment, the students practiced their communication skills in a role play that required them to show moral courage and speak out from a hierarchically inferior position. In another seminar, they discussed their own clinical cases with the dilemma method for moral case deliberation (Molewijk et al. 2008).

\section{Evaluation methodology}

\section{Data collection}

After the interventions were piloted at the different sites, a questionnaire with quantitative and qualitative elements was used to evaluate the advantages and disadvantages of the different interventions. Because we were primarily interested in the continuity of an effect and the transfer to clinical practice (Gollwitzer and Jäger 2009), the evaluation took place several months after each intervention. For example, the participants were asked if they encountered a specific situation in clinical practice that reminded them specifically of the contents in the ethics course. An English questionnaire was drafted allowing all interventions to be rated on a 5-point Likert scale according to recollection of course content, practical usefulness and self-assessed ethical competence (Online Resource 1). A total of 17 questions relating to ethical competence were generated by using a conceptual framework measuring rule abidance, moral sensitivity, moral reasoning and moral motivation and character (De Schrijver and Maesschalck 2013). The questionnaire was then translated into Estonian, Finnish and German and implemented in Survey Monkey (http:/www.surveymonkey.com). Additionally, informal feedback was collected from teachers and group leaders.

\section{Data analysis}

The response rate varied widely between the different subsamples. Overall, 113 midwifery students or midwives responded, but 13 records had to be discarded as being mostly incomplete, leaving a final sample of $n=100$ (age $M=26.0, S D=7.1$ ). Because most intervention-specific subsamples were small $(n<10)$, the analysis was 
conducted only in an exploratory manner. To compare the ratings between different interventions, we conducted multiple t-tests for independent data and adjusted the pvalues with Bonferroni correction. To assess any effects related to aspects of the selfassessed ethical competence subscales, we decided to examine the data on the item instead of the scale level in order to obtain more specific information about potential improvements and remaining difficulties the participants reported. Differences between items in each ethical competence subscale, irrespective of the intervention, were tested with t-tests for dependent data, again followed by Bonferroni correction. Effect sizes are reported with r-values. Correlations to report age-related effects are reported with Kendall's tau, which is more appropriate with small data sets and many tied ranks (Field 2013). All p-values are reported for two-sided testing. In the following results section, we focus on quantitative and qualitative findings that are most informative for the development of our educational model for increasing ethical competence.

\section{Evaluation results}

\section{Intervention ratings}

Turku As expected, the subjects that used the e-learning platform reported having more knowledge about ethical codes (Online Resource 1; item RA1) than participants in any other intervention, $t(21)=-2.43, p=0.024, r=0.47$. In general, it was mentioned that the platform was easy to use and a good supplement to other ethics modules. However, ratings of usefulness for clinical practice (item 6) correlated negatively with age of participants, $r=-0.73, p=0.048$, indicating that the younger students could benefit more from this learning environment. Both the midwives' and the students' discussion groups were rated as being useful for clinical practice. The midwives appreciated discussing clinical issues from a student's point of view, which helped them to support students during their clinical assignments. The students in their group were able to share their feelings and experiences in a safe, peaceful, non-judgmental environment. However, a few participants reported that the sessions were too short to reach a deep level in discussing issues. When compared with the e-learning users, the students in this discussion group tended to report a higher awareness for different perspectives (item MS5), $t(14)=1.91, p=0.077, r=0.45$. Also, older participants tended to report more direct connections to clinical practice (item 7), $r=0.60, p=0.052$.

Tallinn In general, the ratings of usefulness for clinical practice were very high and higher than in other partner institutions, and there was only little variation within and between the interventions. For the ethics seminar in clinical environment, more experienced midwives could make more direct connections to their clinical experience (item 7), $r=0.80, p=0.046$.

Bern The ratings of usefulness for clinical practice (item 6) differed widely between skills-oriented courses and teacher-centered classes. The communication training was rated as being significantly more useful compared to the introductory lecture in ethics $(t(19)=-4.34, p=0.004, r=0.71)$ and the experts' forum on the topic of moral distress $(t(13)=-4.11, p=0.012, r=0.75)$. Also, the one-day seminar that included an in-depth analysis of their own cases using the moral case deliberation method was rated 
significantly higher in terms of practical usefulness compared with these two teaching modules (lecture: $t(35)=3.04, p=0.044, r=0.46$; experts' forum: $t(30)=3.50$, $p=0.015, r=0.54)$. The ratings for the PBL were in an average range.

\section{Self-assessed ethical competence}

Rule abidance Irrespective of intervention and country, the subjects totally agreed that it is very important to know the legal situation and the clinical standards (item RA4), which was rated higher than any other item within this subscale, all $t \mathrm{~s}(57)>4.86$, $p s<0.001, r s>0.54$. They also reported having increased knowledge about ethical codes (item RA1) compared with their self-rated ability in a specific situation to rely on guidelines and codes (item RA3; $t(57)=3.31, p=0.01, r=0.40$ ).

Moral sensitivity Within this subscale, item MS4 was rated lower than any other item; hence they still have trouble generating different solutions in a specific situation, all $t \mathrm{~s}(53)>3.79, p \mathrm{~s}<0.01, r \mathrm{~s}>0.46$. On the other hand, they report now feeling more secure in identifying the ethical aspects of a difficult situation (item MS2) compared to their confidence in their role as a midwife and the corresponding professional responsibilities (item MS1; $t(53)=3.23, p=0.02, r=0.41$ ).

Moral motivation and character Only small and insignificant differences between items were measured for items related to moral motivation and character. Item MC1 received the highest rating, indicating that the students and midwives now more often try to incorporate the consequences for other people involved.

Moral reasoning Finally, the participants now had an increased awareness of the diversity of solutions in a specific situation (item MR4) compared to all other items, all $t \mathrm{~s}(60)>4.05, p \mathrm{~s}<0.001, r \mathrm{~s}>0.46$. The lowest value was measured for item MR2, indicating that they still do not feel very confident making decisions in an ethically difficult situation.

\section{Discussion of pilot and evaluation}

In a pilot at five partner institutions in three countries, we tested the feasibility and effects of 15 interventions. The methodology applied to measure potential effects varied considerably between the different sites and the research design was restricted mainly by organisational and practical reasons. First and foremost, we were not able to implement a pre-post or control group design that would have allowed to measure increases in ethical competence more reliably. Second, the questionnaire we used to measure these effects was not validated and the competences were self-assessed. However, a large set of quantitative and qualitative data including the lecturer's experiences allowed us to draw some important conclusions. E-learning or PBL based cases that are presented with the ICM code of ethics are efficient ways to introduce ethics to inexperienced midwives and students. Ethics days are also a good starting point for familiarizing students with ethical topics when combined with group 
discussions. On the downside, all these interventions are limited in their usefulness for clinical practice as long as they remain abstract and without any personal involvement. Moreover, when e-learning based courses are planned, consideration should be given to easy access and smooth operation, especially when used in the busy clinical environment. This can also be the reason why in our small sample, only the youngest participants rated the electronic platform as useful for clinical practice.

Case discussions in different environments were highly valued by most participants. In Bern, a seminar that included the discussion of personally experienced cases was rated as being much more useful for clinical practice than teacher-centered classes, i.e., when theory was presented without personal involvement. For students, discussing and sharing their own experiences in a safe, non-judgemental environment allow them to express and reflect on their own thoughts, assumptions and values, something they often do not have the courage to do in the clinical environment. Interestingly, more experienced midwives in Tallinn and Turku reported more often making direct links between course contents and their clinical daily routine, which probably reflects the fact that clinical experience facilitates the integration of course contents.

In Bern, the largest effect size was measured for the clinical usefulness of the communication training, where students had to address critical issues with their supervisors in a role play. Although this was the only teaching module that was entirely skills oriented, we assume that this type of ethics course could be an important addition to theory and discussion-based modules.

In a final analysis, we compared moral competence ratings irrespective of intervention in order to create a competency profile of the current sample. The profile we observed shows increased ethical knowledge and awareness compared to aspects of transfer to and application in clinical practice. Subjects reported having increased knowledge of ethical codes and were aware that the legal situation, ethics and clinical standards are important in behaving ethically. In a specific situation however, they had difficulty choosing between a guideline, codes or rules. A similar pattern could be discerned with moral sensitivity; although a facilitated identification of ethical aspects and increased awareness of ethical topics as well as the inclusion of different perspectives could be observed, midwives and midwifery students still had trouble generating a variety of solutions in a specific situation. This makes clear that even increased knowledge and awareness do not guarantee the confidence to make decisions in difficult situations, which is an essential aspect of ethical competence.

\section{The development of an educational model}

\section{General features of the model}

The MidEthics model aimed at increasing ethical competence in students and practicing midwives was developed using the comprehensive exploration results, the lessons learnt from piloting 15 interventions in the educational and clinical environment and the subsequent evaluation. As a starting point, we chose a spiral curriculum that commences in the educational setting and continues into a lifelong learning process (Fig. 1; cf. Leigh et al. 2007). The overarching goal is to promote ethical behavior, which depends on the development of knowledge, skills 
and personal characteristics, such as values, attitudes and traits (Marrelli et al. 2005). At the same time, learning to decide autonomously and to behave in an ethically competent manner requires an iterative revisiting of themes accompanied by growing clinical experience; neither theory nor practice are sufficient by themselves, but have to be interlaced throughout the learning process (Harden and Stamper 1999). Also, new medical and societal developments necessitate continuous learning. With increasing complexity towards the top of the spiral, students and midwives should not only be able to remember course contents but also to apply them skillfully (Harden and Stamper 1999). The spiral is also flexible such that, e.g., mentoring midwives with longer clinical experience can enter the curriculum on the level that meets their needs. Additionally, the salience of each competence is not only dependent on the profession but also on the context, therefore not all levels are equally important in all job settings (Leigh et al. 2007). However, the sequence of levels we have chosen follows a certain logic, as described below, and we believe that some competences are more fundamental than others.

\section{Description of different levels}

Knowledge The experiences from our exploration and pilot phase showed that a common misconception among students is that ethics is something private that can be equated with a personal system of moral values. Initially, students need to understand what ethics is basically about and learn about the purpose of ethical codes,

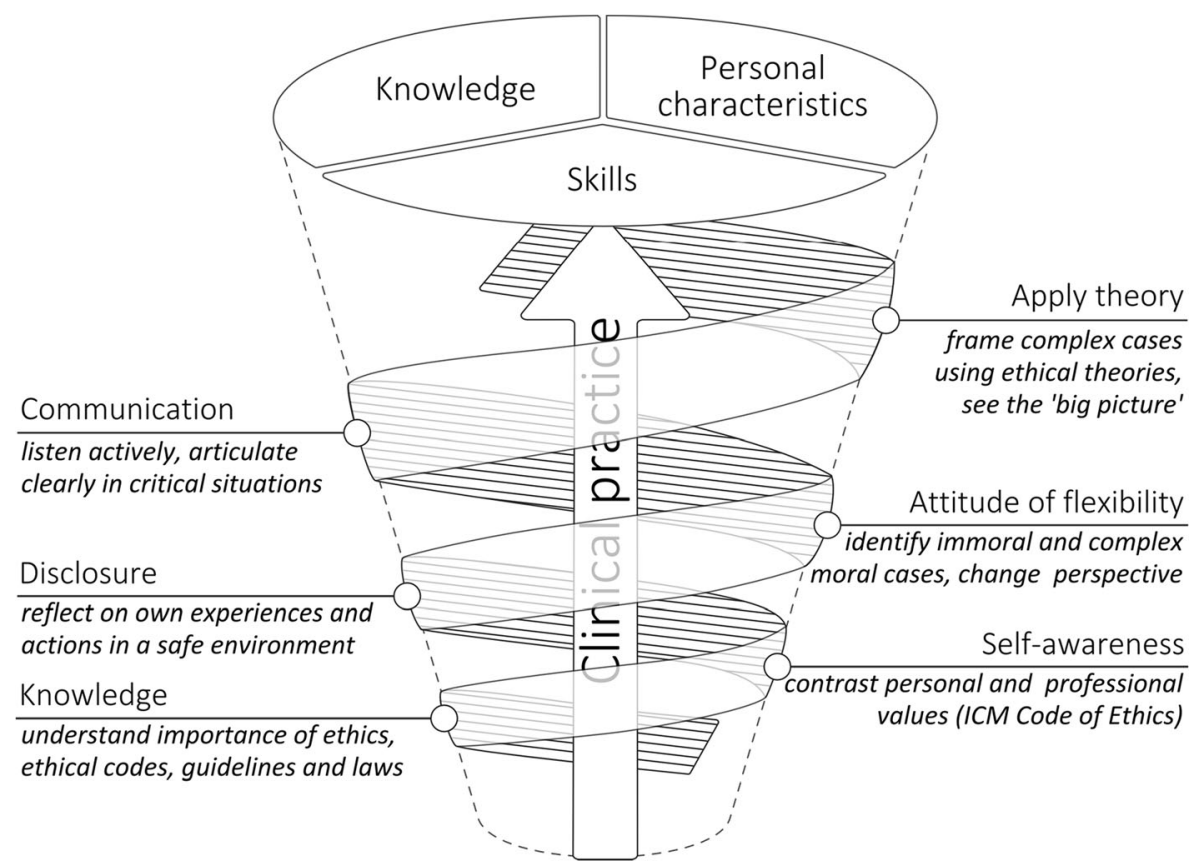

Fig. 1 The MidEthics model, a spiral curricular model representing different stages of increasing ethical competence 
guidelines and laws. Ethics days and lectures seem to be a good starting point to raise awareness on ethics; however they should not comprise much ethical theory on this level, as this often remains too abstract without a direct link to clinical practice.

Self-awareness While critical reflection on one's own behavior is an essential part of professional competence in general (Kulju et al. 2015), midwifery students should learn to distinguish between personal and professional values. Tutored PBL or blended learning sessions allow the presentation of everyday ethical problems that are then discussed in groups, and in this process, the ICM code of ethics is a good resource for participant guidance.

Disclosure The midwifery students who were interviewed in our exploration reported a feeling of powerlessness because they felt they had limited influence on procedures in the clinical setting. Many students and younger midwives also feel uncertain because they realize that there is a gap between theory and practice (Cohen and Erickson 2006). Therefore, they should reflect on their own experiences together with their mentoring midwives or peers, recognize conflicting values, disclose their thoughts in a safe environment and share their concerns and uncertainties (Cohen and Erickson 2006; Wilson 2008). These guided discussion groups should be small enough to provide space for individual self-reflection and examination of one's own decisions and actions for potential bias (Foster and Lasser 2010).

Attitude of flexibility and communication In the clinical context, midwives also need an attitude of flexibility or openness. Every woman or family has its individual needs, values and preferences. A midwife should try to respect these values in a non-judgmental way. When generating solutions for a specific problem, the ethically competent midwife considers the perspective of all persons involved and does not always stick to a single rule or guideline (De Schrijver and Maesschalck 2013). On the other hand, being tolerant or flexible also has limits; midwives need to differentiate between cases of moral wrongness and those of moral complexity (cf. ch. 2.1; Monteverde 2014). This comprises being morally sensitive to violations of ethical guidelines and professional values and acting accordingly. In morally challenging situations, these steps typically require advanced communication skills. Listening actively to understand the perspective of others and clearly articulating the core of a problem is essential to solve problems. Especially inexperienced midwives or students should be trained specifically to talk to their supervisor in cases of moral wrongness in order to avoid misbehavior.

Apply theory With increasing clinical experience and responsibility, midwives are typically not only able to decide autonomously, but are also more involved in interdisciplinary decisions, either in informal discussions or formal ethics meetings or committees (Cusveller and Schep-Akkerman 2016). Complex cases need to be framed with the help of ethical theories to recognize the core of the conflict. In this case, all health professionals involved also need to see the 'big picture' of the problem and should also tolerate values that are distinct for other professions. 


\section{Overall conclusions}

In this international project, we developed a set of interventions to increase ethical competence in midwives on the local level and created a higher-level model for education and practice. Because our model is based on different competences, it can be applied in different contexts, where some competences may be more important than others. Most likely, other health professionals will also go through similar steps in order to increase their ethical competence, although this depends on their position within an organization and their tasks and responsibilities. While the model is flexible in terms of contextual adaptability, the downside is that we did not include any concrete pedagogical guidelines on how to increase ethical competence, as this depends even more on the context, the available resources, the existing curriculum, and clinical peculiarities. However, it is clear that theory and practice should be intertwined whenever possible. While this may sound trivial, many curricula still include theoretical lectures and seminars even before the students' first clinical assignment. It is important that students understand the purpose of ethics in daily practice, but contrasting different ethical theories could strengthen the students' view that ethical decisions are somehow arbitrary and are grounded on their personal convictions. Also, this could lead to perceiving ethics as something abstract, complex and therefore irrelevant for clinical practice. It is interesting to note that in some seminars, we observed more experienced midwives making more direct connections to their daily practice. This highlights that some topics may come too early in order to be learnt and integrated, but it also shows that experienced midwives benefit from these discussion groups too. Overall, more research should be undertaken on the efficiency of different pedagogical forms of teaching, and more skills-oriented teaching should be implemented, especially for less experienced midwives, in order to be useful in clinical practice.

Acknowledgements We acknowledge the support of PD Dr. Rouven Porz for helpful comments in the exploration phase, Dr. Settimio Monteverde for invaluable comments on the development of interventions in Bern, Dr. Joca Zurc for external project evaluation and Ira Polyanskaya for designing the spiral model.

This project has been funded with the support of the Lifelong Learning Programme of the European Union (Project Code 540082-LLP-1-2013-1-FI-ERASMUS-EQR). This publication reflects the views only of the authors, and the Commission cannot be held responsible for any use which may be made of the information contained therein.

\section{Compliance with ethical standards}

Conflict of interest statement The authors declare that there is no conflict of interest.

\section{References}

American Association of Critical-Care Nurses. 2004. The 4 A's to rise above moral distress. Aliso Viejo, CA: American Association of Critical-Care Nurses.

Barandun Schäfer, U., A. Ulrich, B. Meyer-Zehnder, and I.A. Frei. 2015. Acute care nurses' ethical reasoning: a thematic analysis. Pflege 28(6): 321-327. doi:10.1024/1012-5302/a000457.

Bolmsjö, I.A., L. Sandman, and E. Andersson. 2006. Everyday ethics in the care of elderly people. Nursing Ethics 13(3): 249-263. 
Cahill, Heather A. 2001. Male appropriation and medicalization of childbirth: an historical analysis. Journal of Advanced Nursing 33(3): 334-342. doi:10.1046/j.1365-2648.2001.01669.x.

Cohen, Jeryl S., and Jeanne M. Erickson. 2006. Ethical dilemmas and moral distress in oncology nursing practice. Clinical Journal of Oncology Nursing 10(6): 775-782. doi:10.1188/06.cjon.775-780.

Cusveller, Bart, and Annemiek Schep-Akkerman. 2016. Towards a competency assessment tool for nurses in ethics meetings. Nursing Ethics 23(4): 413-420. doi:10.1177/0969733014567170.

De Schrijver, Annelies, and Jeroen Maesschalck. 2013. A new definition and conceptualization of ethical competence. In Achieving ethical competence for public service leadership, ed. D. Menzel and T. Cooper, 29-50. Armonk, NY: M.E. Sharpe.

Field, Andy. 2013. Discovering statistics using IBM SPSS Statistics. London: Sage.

Foster, Illysa R., and Jon Lasser. 2010. Professional ethics in midwifery practice. Sudbury, MA, USA: Jones \& Bartlett Publishers.

Gallagher, Ann. 2006. The teaching of nursing ethics: content and method. In Essentials of teaching and learning in nursing ethics: perspectives and methods, ed. A. Davis, V. Tschudin, and L. DeRaeve, 223239. London, UK: Churchill Livingsone.

Gollwitzer, Mario, and Reinhold S. Jäger. 2009. Evaluation kompakt. Beltz Verlagsgruppe: Weinheim.

Harden, R.M., and N. Stamper. 1999. What is a spiral curriculum? Medical Teacher 21(2): 141-143. doi: $10.1080 / 01421599979752$.

International Confederation of Midwives. 2014a. International code of ethics for midwives.

International Confederation of Midwives. 2014b. Philosophy and model of midwifery care.

Kavathatzopoulos, Iordanis. 2003. The use of information and communication technology in the training for ethical competence in business. Journal of Business Ethics 48(1): 43-51. doi:10.1023 /b:busi.0000004366.08853.72.

Kulju, Kati, Minna Stolt, Riitta Suhonen, and Helena Leino-Kilpi. 2015. Ethical competence: a concept analysis. Nursing Ethics. doi:10.1177/0969733014567025.

Lachman, Vicki D. 2007. Moral courage: a virtue in need of development? MEDSURG Nursing 16(2): 131133.

Leigh, Irene W., I. Leon Smith, Muriel J. Bebeau, James W. Lichtenberg, Paul D. Nelson, Sanford Portnoy, Nancy J. Rubin, and Nadine J. Kaslow. 2007. Competency assessment models. Professional Psychology: Research and Practice 38(5): 463-473. doi:10.1037/0735-7028.38.5.463.

Liaschenko, J., N.Y. Oguz, and D. Brunnquell. 2006. Critique of the "tragic case" method in ethics education. Journal of Medical Ethics 32(11): 672-677. doi:10.1136/jme.2005.013060.

Lindqvist, Martti. 2005. Sosiodraama matkana yhteisön kollektiiviseen alitajuntaan [Sociodrama as a journey to the collective subconscious of society]. In Miten käytän toiminnallisia menetelmiä? ed. T. Janhunen and S. Sura, 9-27. Tampere: Resurssi.

Marrelli, Anne F., Janis Tondora, and Michael A. Hoge. 2005. Strategies for developing competency models. Administration and Policy in Mental Health and Mental Health Services Research 32(5): 533-561. doi:10.1007/s10488-005-3264-0.

Marshall, Jayne E. 2005. Autonomy and the Midwife. In Decision making in midwifery practice, ed. Maureen D. Raynor, Jayne E. Marshall, and Amanda Sullivan, 9-21. London, U.K.: Elsevier.

McLaughlin Dorcas, E., E. Freed Patricia, and A. Tadych Rita. 2006. Action methods in the classroom: creative strategies for nursing education. International Journal of Nursing Education Scholarship 3(1): 118. doi:10.2202/1548-923X.1229.

Molewijk, A.C., T. Abma, M. Stolper, and G. Widdershoven. 2008. Teaching ethics in the clinic. The theory and practice of moral case deliberation. Journal of Medical Ethics 34(2): 120-124. doi:10.1136 /jme.2006.018580.

Monteverde, Settimio. 2014. Undergraduate healthcare ethics education, moral resilience, and the role of ethical theories. Nursing Ethics 21(4): 385-401. doi:10.1177/0969733013505308.

Noseworthy, D. Ann, R. Phibbs Suzanne, and A. Benn Cheryl. 2013. Towards a relational model of decisionmaking in midwifery care. Midwifery 29((7): e42-e48. doi:10.1016/j.midw.2012.06.022.

Olsen, Ole, and Jette A. Clausen. 2012. Planned hospital birth versus planned home birth. Cochrane Database of Systematic Reviews (9). doi:10.1002/14651858.CD000352.pub2.

Thompson, Anne. 2004. Bridging the gap: teaching ethics in midwifery practice. The Journal of Midwifery \& Women's Health 49(3): 188-193. doi:10.1016/s1526-9523(04)00105-9.

Wilson, J. 2008. Bridging the theory practice gap. Australian Nursing and Midwifery Journal 16(4): 25. 